Please quote as: Thillainathan, N.; Hoffmann, H.; Hirdes, E. M. \& Leimeister, J. M. (2013): Enabling Educators to design Serious Games - A Serious Game Logic and Structure Modeling Language. In: 8th European Conference on Technology Enhanced Learning (EC-TEL), Paphos, Cyprus. 


\title{
Enabling Educators to design Serious Games - A Serious Game Logic and Structure Modeling Language
}

\author{
Niroshan Thillainathan, Holger Hoffmann, Eike M. Hirdes, Jan Marco Leimeister \\ Kassel University, Information Systems, Kassel, Germany \\ \{thillainathan, hhoffman, hirdes, leimeister\}@uni-kassel.de
}

\section{Introduction}

Serious games are applications combining educational content with gameplay by integrating learning objectives into a game-like environment to keep up the player's motivation to continue playing, and hence learning. This characteristic is highly sought after in educational contexts, making serious games a big asset for didactics [1]. Offering new learning contents through a game not only induces higher motivation, employing serious games can also yield higher learning success than presenting material in a classical, non-computer based, way [2]. Only few people having the proper didactical background to tailor the learning objectives to the students' need also have the programming knowledge and game design skills allowing them to develop didactically and technically sound serious games [3, 4]. In this paper, we argue for an approach to enable didactical experts, i.e. educators, to develop serious games adapted to their own learning content. To address this problem we develop a tool allowing educators to visually design their serious games, which is based on model driven development techniques that allow the generation of software from visual models. We describe the first step towards this tool, the development of the underlying domain specific modeling language (DSML).

\section{GLiSMo: Serious Game Logic and Structure Modeling Language}

To identify requirements for the development of our domain specific modeling language GLiSMo an in-depth literature review was conducted. As the result of our review, we have identified 18 publications mentioning 23 influencing factors for serious games, from which we have derived 24 requirements. GLiSMo is a modeling language, which allows designing the structure as well as the logic of a serious game.

Serious game structure modeling describes how a serious game and its game world are built. Here, the Serious Game Root element, present once for each game modeled, plays a special role, as it is the point of origin for all other elements. Serious game root elements can have one or more acts, which have similarities to levels in common games

Parts of this research were funded by the German Federal Ministry of Education and Research and the European Social Fund in the project BlendedContENT (www.blendedcontent.de), FKZ 01PF08022A

adfa, p. 1, 2011

(C) Springer-Verlag Berlin Heidelberg 2011 
and are divided by one or more scenes. A scene describes a specific spot or place within the game world. This can for example be a room within a building. The element Object represents all kind of objects within the game world, i.e. doors, boxes, trees. The Character element represents the player itself and non-player characters (NPC). An inventory element is used for storing objects taken by the player. The element feedback manager manages the textual feedback given to the player. The Reward Manager stands for the scoring and rewarding within a serious game. The Audio Manager and Video Manager allow the playback of audio and video during the game. The GUI Manager is used to display buttons, textboxes, scores or the game menu.

Serious game logic modeling characterizes the behavior in terms of how does the game react on specific actions performed by the player, or events occurring during game play. It also describes the assessment of player actions and the game adaption according to the adaption results. To define the boundaries of a logic model, we denote the initial-state by a black circle; final-states are represented by an encircled black circle. The element action enables the player to interact with the game world. This has different types, e.g. take object, use object, representing different interaction methods in the game world. The element task represents different tasks, i.e. multiple-choice questions. The associated assessment element evaluates the result and initiates processes to give feedback and achievements to the player. To manage the information and control flow, we use so called streams to connect the elements. To make a progress along the control flow the player must execute the described actions. To send events and messages between elements a dotted arrow will be used. Furthermore fork and join as well as branch elements are used to manage parallel and branched flows.

\section{Conclusion and Future Work}

Supporting educators design serious games through model driven development techniques is a promising approach. In this paper we proposed our first steps towards a DSML for serious games. Future work includes the extension of the modeling language with more elements supporting different tasks and assessment methods. Furthermore GLiSMo must be evaluated with educators to determine its usability. In parallel a visual design tool for GLiSMo will be implemented.

\section{References}

1. Brennecke, A. and H. Schumann. A General Framework for Digital GameBased Training Systems. in IADIS International Conference Game and Entertainment Technologies. 2009. Algarve, Portugal.

2. Pivec, M., Editorial: Play and learn: potentials of game-based learning. British Journal of Educational Technology, 2007. 38(3): p. 387-393.

3. Chandler, H.M., The Game Production Handbook. 2nd ed. 2010, Ontario: Jones \& Bartlett Publishers.

4. Torrente, J., et al. 〈e-Adventure〉: Introducing educational games in the learning process. in Education Engineering (EDUCON). 2010. 